\title{
Implementation of Traffic E-Government Cloud Technology for Department of Transportation
}

\author{
Jian Lian \\ Department of EEIT \\ Shandong University of Science and Technology \\ Jinan, China \\ e-mail: lianjianlianjian@163.com \\ Yan Zhang \\ Department of EEIT \\ Shandong University of Science and Technology \\ Jinan, China \\ e-mail: clearawz@163.com
}

\author{
Mingqu Fan \\ Department of EEIT \\ Shandong University of Science and Technology \\ Jinan, China \\ e-mail: fmq@sdust.eud.cn \\ HaiTao $\mathrm{Pu}$ \\ Department of EEIT \\ Shandong University of Science and Technology \\ Jinan, China \\ e-mail: kdpht@163.com
}

\begin{abstract}
Transport is one of most important aspect of economy and social development in one country. For recent years transportation industry in China has made plenty of great achievements, in which the transportation industry as well as Department of Transportation contributed greatly. Gradually public services have brought more pressures than before, especially some of the new pressures coming from transportation capability, green travel, transportation safety emergency security, convenient public travel service. The transportation industry information has much to do with following systems, such as the e-government system, decision support system, industry regulation system, safety emergency security system, public service system, which are all included in the management system of Department of Transportation in China.
\end{abstract}

Keywords-Transportation information ;Traffic e-government cloud; Cloud computing

\section{INTRODUCTION}

Traffic Transportation Information construction, which plays an important role in the Traffic Industry is the substantial foundation of traffic industry development ${ }^{[1]}$. Hence, "To Raise the Intelligent and Modern level of Traffic Transportation totally through implementing a batch of important information projects during the 12th Five-Year Plan" has been proposed in Development planning of traffic transportation information construction during the 12th Five-Year Plan ${ }^{[2]}$.

In the history of traffic transportation information construction process, firstly the China Transportation Information Network was built with promotion of the "Golden Traffic" program ${ }^{[3]}$, then plenty of relative traffic transportation information systems are applied by the Departments of Plan, Management, and Transportation ${ }^{[4]}$. Till now there are two layers of Data Center in Ministry and Province, forty-one traffic bone networks, and industrial data centers, private wide area networks. All the results show that we have made brilliant achievements in the last years, but we must be aware that there are plenty of problems need to be solved in the future ${ }^{[5]}$

\section{The Establishment Process of Traffic E- GOVERNMENT CLOUD}

Virtualization is the foundation of Traffic E-Government, and with virtualization we can implement the abstraction, standardization, and resource pooling of Traffic EGovernment IT infrastructure facility, which makes the users to neglect the specific details of the hardware. Based on Virtualization Traffic E-Government cloud can supply with accurate prompt traffic travel information service, overall logistics platform service for cross-region and cross-section integrated application of the whole industry, the public, and enterprises $^{[6]}$.

With the development towards Cloud Computing, Traffic E-Government changes the original construction pattern and needs transient time ${ }^{[\rceil]}$. Meanwhile, Cloud Computing, which is one complex systematical engineering, evolves from IaaS to PaaS and SaaS.

(1) Phase 1: To Build the IaaS Platform

Firstly we build the Traffic E-Government infrastructure Cloud Platform, that is IaaS Platform, which would be used to deploy the new systems. For example, the undergoing and implementing systems, such as "Highway and Waterway Security Smooth and Emergency Response System", "Highway and Waterway Traffic Travel Information Service System", "Highway and Waterway Construction and Transportation Market Credit Information Service System", and "Traffic Transportation Operation Monitoring Alarm and Decision Analysis System", which all should follow the IaaS pattern to construct infrastructure resource platform.

(2) Phase 2:To Deploy the Business Systems

In this phase, we mainly complete the Traffic EGovernment Cloud Platform service improvement, gradually deploy the necessary business systems in the platform and migrate the old systems to the platform. Meanwhile, we also should expand the IaaS part according to the business loading situation of the cloud platform. 
(3) Phase 3: To Construct the PaaS and SaaS

In the last phase, we need to analyze and abstract the common features of every business and form the Traffic EGovernment Cloud Computing PaaS and SaaS layers with the gradual development of the Traffic E-Government Cloud Platform Business Systems. At the same time, combining with the deployment and maintenance experience, we can standardize and formulate the Traffic E-Government Cloud Platform operation pattern, architecture, service methods and even business pattern. In this phase, Traffic E-Government Cloud Platform have been constructed completely, and it can serve the traffic transportation industry already.

\section{IMPLEMENTATION OF TRAFFIC E-GOVERNMENT}

\section{A. Platform Framework of Traffic E-Government Cloud IaaS}

As shown in Fig 1, Traffic E-Government Cloud Platform Framework is consist of IaaS Layer, Business Application Layer, User Layer, and Security Management Module, in which the IaaS is mainly responsible for deploying the Server, Storage and other Networking Hardware, automatically manage the hardware resource pooling through Virtualization, and arrange the resource pool for the Cloud Service Management Platform; Business Application Layer supplies with plenty of Application systems for Traffic E-Government based on Traffic basic Database; Security Management Module supplies with the End-to-End Protection for IaaS Layer and Business Application Layer; User Layer divides the industrial users and public users according to the business requirement of the different users. The industrial users accept corresponding service through private WAN and the public users accept the cloud Service through Internet.

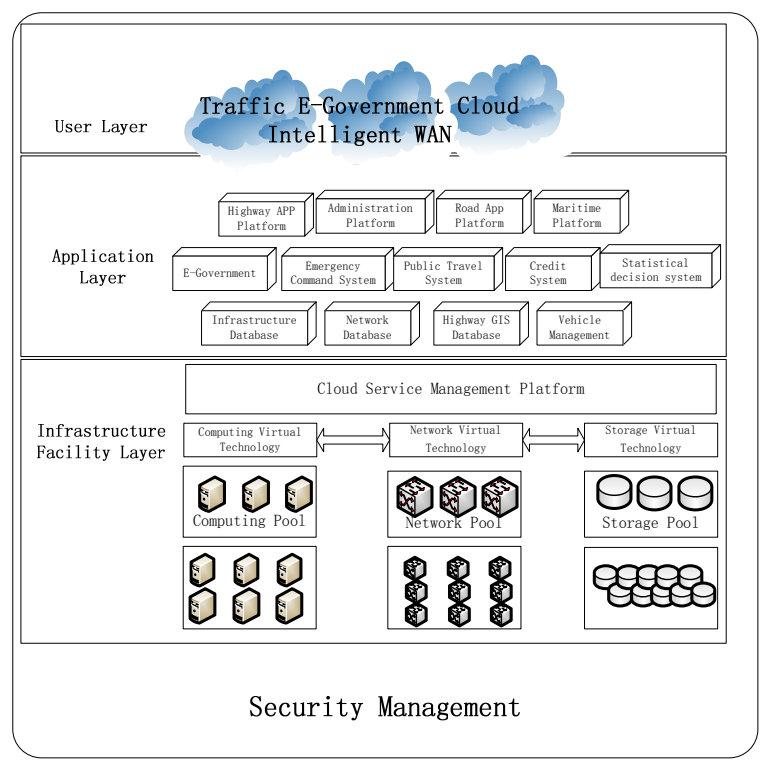

Fig1.The architecture of Traffic E-Government cloud IaaS

\section{B. The Construction of the Infrastructure Facility and Resource Pooling}

(1) To Construct the Computing Resource Pool

We recommend that the servers used in Traffic EGovernment Cloud Platform should adopt $\mathrm{x} 86$ architecture to compose the Virtualized Computing Resource Pool, according to the requirement of "Emergency Command System", "Public Travel System", "Credit Information System", and "Decision Analysis System" we determined the quantity of the physical servers, which we virtualized to form the Computing Resource Pool through Virtualization software, such as H3C CVK. Finally all the systems would operate in the virtual machines of the computing resource pool.

(2) To Construct the Storage Resource Pool

Through the abstraction to storage hardware and the transparency to eliminate the complexity of the software/hardware system in bottom layer with virtualization technology, we can form the storage resource pool. And with the resource and the unified user interface we can manage and control the data, simplify the storage allocation and management process, which realizes the seamless migration of the files among virtual machines.

(3) To Construct the Network Resource Pool

The Construction of Traffic E-Government Cloud Platform needs Cloud Computing Data Center equipments, which can perform the monitoring to Virtual Machines. With the identification of virtual machines, it can supply with reliable Security Protection among the virtual machines to satisfy classified protection requirement of traffic industry.

\section{To Construct the Cloud Management Platform}

Cloud Management Platform can be used to implement the Traffic E-Government Cloud Infrastructure integrated, intelligent, visible and automatic Management, the fully automatic Service for the upper Business Application Layer, and automatic supply and business for IT service, such as management of the cloud business workflow.

\section{Deployment}

In the deployment process, we should build the security protection architecture with virtualization as the technology support. For example, the firewall and other security devices all need to support virtualization. And different users can be mapped to different virtualization instances based on VLAN, in which every instance has independent security control strategy and independent management task. In the Traffic EGovernment cloud security deployment, we should allocate independent virtual firewall instance and realize the security protection strategy deployment among the virtual machines through networking devices, identifying the internal virtual machine throughput, and the integrated security adapters inside the devices.

\section{CONCLUSION}

Traffic E-Government Cloud is the supporting platform based on Internet of Things, Big Data Analysis and other new techniques. It contributes so much to realize the Internet of Vehicles, which can help to implements People, Vehicle, 
and Road Cooperation with RFID and WSN. With advanced Sensor Technology, Data Communication Technology we would acquire real-time, dynamical traffic status information, and Traffic E-Government supplies for the analysis of data with powerful computing platform. We can get key data through analyzing the Big Data, optimize and configure the traffic transportation resources, analyze and predict the traffic status. And it can be foreseen that the service of traffic transportation industry would be more intelligent and humanized.

\section{ACKNOWLEDGMENT}

This work has been supported by the Shandong Province Higher Educational Science and Technology Program (J10LG16 and J11LG21), the Shandong Science and the Technology Development Project (2012GGX10122, 2013G0021109), the Shandong Province Higher Educational Science and Technology Program (J11LG21, J13LN35), Jinan Science and Technology Development Project (201202058), Teaching Reform of the Key Project of Shandong Province (2012073,2012552,2012033) and International Cooperation Program for Excellent Lecturers by Shandong Provincial Education Department, and Teaching Research Project of Shandong University of Science and Technology (qx2013286)and the Shandong Doctoral Foundation under Grant BS2009DX039.

\section{REFERENCES}

[1] National Institute of Standards and Technology. About NIST[EB/OL]. (2012-4-18)[2012-9-7].

[2] ISO. JTC 1/SC 27 IT Security techniques[EB/OL]. (2012-6-8)[20129-2].

[3] HuiLi Wang, Chen Yang . Cloud Computing Security and Standard Research [J]. Information Technology and Informatization, 2012(5) : $18-21,20$.

[4] ISO. ISO/IEC WD TS 27017[EB/OL]. (2012-5-8)[2012-9-3].

[5] ISO. ISO/IEC WD TS 27018[EB/OL]. (2012-4-8)[2012-9-4].

[6] National science library of Chinese academy of science. Science Research Dynamical Monitoring [EB/OL]. (2012-7-30)[2012-9-5].

[7] Information website of the Chinese academy of sciences. Information technology, Chinese academy of sciences, research and application [EB/OL]. (2011-9-30)[2012-9-6]. 